\title{
GRAVITATIONAL WAVES AND THE MAXIMUM SPIN FREQUENCY OF NEUTRON STARS
}

\author{
Alessandro Patruno, Brynmor Haskell, and Caroline D’ Angelo \\ Astronomical Institute "Anton Pannekoek," University of Amsterdam, Postbus 94249, NL-1090 GE Amsterdam, The Netherlands \\ Received 2011 September 1; accepted 2011 November 16; published 2012 January 19
}

\begin{abstract}
In this paper, we re-examine the idea that gravitational waves are required as a braking mechanism to explain the observed maximum spin frequency of neutron stars. We show that for millisecond X-ray pulsars, the existence of spin equilibrium as set by the disk/magnetosphere interaction is sufficient to explain the observations. We show as well that no clear correlation exists between the neutron star magnetic field $B$ and the X-ray outburst luminosity $L_{X}$ when considering an enlarged sample size of millisecond X-ray pulsars.
\end{abstract}

Key words: gravitational waves - pulsars: individual (SAX J1808.4-3658, XTE J1814-338, IGR J00291+5934, XTE J1751-305)

\section{INTRODUCTION}

Some accreting neutron stars (NSs) in low-mass X-ray binaries are thought to be spun up by transfer of angular momentum from an accretion disk. The spin-up process brings several NSs to spin in the millisecond range. We refer to these systems as millisecond X-ray pulsars (MXPs), which comprise accretion-powered millisecond pulsars (with pulsations formed via magnetic channeled accretion) and nuclear-powered millisecond pulsars (with burst oscillations observed during thermonuclear bursts). These are very old systems with lifetimes of several billion years (van Paradijs \& White 1995).

The spin frequency $\left(v_{s}\right)$ of MXPs has been measured to date in 22 systems (Patruno 2010a; Papitto et al. 2011a) with a range between $\approx 182 \mathrm{~Hz}$ and $\approx 620 \mathrm{~Hz}$. The narrow range of spin frequencies appears surprising considering that the binary lifetime is a billion years whereas the spin-up process operates on timescales of $10^{7}-10^{8} \mathrm{yr}$ (White et al. 1988).

Chakrabarty et al. (2003) and Chakrabarty (2008) observed that the spin-frequency distribution of these systems (in 2003 comprising only 11 systems) was consistent with a uniform distribution with a cutoff frequency of $730 \mathrm{~Hz}$. Patruno (2010a) has shown how the same cutoff is found today with a sample size that is twice as large. The existence of a cutoff at such a relatively low frequency is somewhat unexpected because the Keplerian break-up frequency for a typical NS with any realistic equation of state is well above the $730 \mathrm{~Hz}$ cutoff.

White \& Zhang (1997, henceforth WZ97) argued that the tight range of spin periods over two orders of magnitude in observed luminosity could be explained if these systems had reached spin equilibrium. In this picture the stellar magnetic field truncates the disk close to or at the corotation radius (where the stellar spin frequency is equal to the Keplerian frequency of the disk), and no net angular momentum is transferred onto the star.

This interpretation leads to an unexpected correlation between the NS magnetic field at equilibrium $B$ and the outburst X-ray luminosity $L_{X}$. Indeed in transient systems $L_{X}$ is given by the average mass accretion rate during an outburst, which depends on the outburst length and on the outburst recurrence time. These quantities are set solely by the accretion disk physics (Lasota 2001), so it seems difficult to justify a correlation with the magnetic field.

Following an earlier suggestion of Papaloizou \& Pringle (1978) and Wagoner (1984), Bildsten (1998) proposed a different mechanism to limit the spin-up of MXPs, introducing a loss of angular momentum via gravitational wave $(\mathrm{GW})$ emission. In this scenario a correlation between $B$ and $L_{X}$ is much weaker and a sharp spin-frequency cutoff is expected due to the strong spin-frequency dependence $\left(\propto v_{s}^{5}\right)$ of the spindown torque.

However, recent observational results on some MXPs have shown that the efficiency of GW-induced spin-down for MXPs might be very low. Patruno (2010a), Hartman et al. (2011), and Papitto et al. (2011b) measured the long-term evolution of the spin frequency of IGR J00291+5934, revealing both accretion torques during one outburst and a long-term spin-down during quiescence, which they interpreted as due to magneto-dipole torques. The combined effect of spin-up in outburst and spindown in quiescence leads to a timescale of several billion years for the evolution of the $599 \mathrm{~Hz}$ spin frequency of this MXP (Patruno 2010a). Hartman et al. (2011) and Papitto et al. (2011b) also showed that GW torques are unimportant for MXPs with frequencies up to $599 \mathrm{~Hz}$.

Another recent result (Hartman et al. 2008, 2009; Haskell \& Patruno 2011) showed that in MXP SAX J1808.4-3658 and XTE J1814-338 stringent upper limits can be set on the accretion-induced spin-up of the NS. Haskell \& Patruno (2011), in particular, rejected the scenario in which a continuous train of GWs is responsible for balancing the spin-up process. Those authors proposed spin equilibrium as set by the disk/magnetosphere interaction as the most likely mechanism to explain the observed behavior of the two MXPs.

In this paper, we revisit the analysis of WZ97 and show that:

1. the observations suggest that a large majority of MXPs can be in spin equilibrium without implying a correlation between the $B$ field and the X-ray outburst luminosity;

2. invoking GWs to explain the cutoff in the spin distribution is not necessary given the uncertainty in models of more realistic disk/magnetosphere interactions.

\section{MILLISECOND X-RAY PULSAR SAMPLE}

We use 18 MXPs (see Table 1) which have both confirmed spin frequencies (see Patruno 2010b, and references therein) and a realistic determination of the outburst flux (see Watts et al. 2008; Riggio et al. 2011b).

We do not use any accreting NS whose $v_{s}$ is inferred only from observations of twin-kHz quasi-periodic oscillations (QPOs). The idea that the separation $\Delta v$ between twin-kHz QPOs is 
Table 1

Millisecond X-Ray Pulsar Sample with Spin Frequency and Luminosity

\begin{tabular}{|c|c|c|c|c|}
\hline Source Name & $\begin{array}{c}L_{X} \\
\left(10^{36} \mathrm{erg} \mathrm{s}^{-1}\right)\end{array}$ & $\begin{array}{l}\text { Spin Freq. } \\
(\mathrm{Hz})\end{array}$ & $\begin{array}{c}\text { Outb. Flux } \\
\left(10^{-8} \mathrm{erg} \mathrm{s}^{-1} \mathrm{~cm}^{-2}\right)\end{array}$ & $\begin{array}{l}\text { Distance } \\
(\mathrm{kpc})\end{array}$ \\
\hline 4U 1608-522 & 40 & 620 & 2.0 & $4.1(0.4)$ \\
\hline Aql X-1 & 32 & 550 & 1.3 & $4.55(1.35)$ \\
\hline SAX J1748.9-2021 & 31 & 442 & 0.4 & $8.1(1.3)$ \\
\hline KS 1731-260 & 30 & 524 & 0.49 & $7.2(1)$ \\
\hline XTE J1751-305 & 28 & 435 & 0.29 & $9(3)$ \\
\hline IGR J17191-2821 & 18 & 294 & 0.26 & $7.5(3.5)$ \\
\hline IGR J17511-3057 & 15 & 244 & 0.2 & $8(2)$ \\
\hline MXB 1659-298 & 13 & 567 & 0.08 & $12(3)$ \\
\hline XTE J0929-314 & 7 & 185 & 0.1 & $7.8(4.2)$ \\
\hline SAX J1750.8-2900 & 7 & 601 & 0.12 & $6.79(0.14)$ \\
\hline GRS 1741.9-2853 & 6 & 589 & 0.1 & $7.2(2.8)$ \\
\hline XTE J1807-294 & 6 & 191 & 0.072 & $8.35(3.65)$ \\
\hline SAX J1808.4-3658 & 5 & 401 & 0.35 & $3.5(0.1)$ \\
\hline IGR J00291+5934 & 5 & 599 & 0.16 & $5(1)$ \\
\hline XTE J1814-338 & 4 & 314 & 0.069 & $6.7(2.9)$ \\
\hline SWIFT J1756.9-2508 & 3 & 182 & 0.04 & $8(4)$ \\
\hline HETE J1900.1-2455 & 2 & 377 & 0.09 & $4.7(0.6)$ \\
\hline NGC $6440 \mathrm{X}-2$ & 2 & 206 & 0.019 & $8.1(1.3)$ \\
\hline
\end{tabular}

directly related with $v_{s}$ was supported by the observed ratio $\Delta v / v_{s} \approx 0.5-1.0$ in a few MXPs (van der Klis et al. 1996; Wijnands et al. 2003). It has recently been shown, however, that twin-kHz QPOs might not be a good proxy for NS spin frequencies and could even be completely unrelated to $v_{s}$ (Méndez \& Belloni 2007; Yin et al. 2007).

In comparison, WZ97 used a sample of ten accreting NSs, five of which had spin frequencies inferred only from the observation of twin-kHz QPOs and five from burst oscillations.

\section{DISK/MAGNETOSPHERE INTERACTION}

The interaction between a star's magnetic field and the surrounding accretion disk provides a natural mechanism to limit the spin frequency of the star. By assuming that most systems have reached their limiting spin frequency, WZ97 estimated the magnetic field strength for the sources in their sample and found an unexpected correlation between $B$ and $L_{X}$.

For strong magnetic fields, the disk will be truncated at some distance from the star (at the magnetospheric radius, $r_{m}$ ), which is generally estimated to be (Pringle \& Rees 1972)

$$
r_{m}=35 \xi \dot{M}_{-10}^{-2 / 7} M_{1.4}^{-1 / 7} R_{10}^{12 / 7} B_{8}^{4 / 7} \mathrm{~km},
$$

where $\dot{M}_{-10}$ is the accretion rate in units of $10^{-10} M_{\odot} \mathrm{yr}^{-1}, M_{1.4}$ is the NS mass in units of $1.4 M_{\odot}, R_{10}$ is its radius in units of $10 \mathrm{~km}$, and $\xi$ parameterizes the uncertainties in evaluating the torque at the edge of the accretion disk (thought to be in the range $\xi \approx 0.3-1$; Psaltis \& Chakrabarty 1999).

Inside $r_{m}$ gas will be channeled onto the star, spinning the star up at a rate

$$
\dot{J}=\dot{M} \sqrt{G M r_{m}} \text {. }
$$

However, if the star is spinning very fast, the disk may be truncated outside the corotation radius $r_{c}$, where the Keplerian frequency is equal to the spin rate of the star:

$$
r_{\mathrm{co}}=1683 M_{1.4}^{1 / 3} v_{s}^{-2 / 3} \mathrm{~km},
$$

and the spinning magnetosphere will present a centrifugal barrier that can inhibit accretion (the so-called propeller regime;
Illarionov \& Sunyaev 1975). This suggests that the star will eventually spin at a rate such that $r_{m} \simeq r_{\mathrm{co}}$ ("spin equilibrium").

Under this assumption, the magnetic field at equilibrium can be estimated as

$$
B=8.8 \times 10^{10} \xi^{-7 / 4} \dot{M}_{-10}^{1 / 2} M_{1.4}^{5 / 6} R_{10}^{-3} v_{s}^{-7 / 6} \mathrm{G} .
$$

The 10 accreting NSs used by WZ97 showed a spread in luminosities over two orders of magnitude whereas the spin periods clustered between 2.8 and $3.8 \mathrm{~s}(263-362 \mathrm{~Hz})$. Equation (4) shows that the small range of spin periods over a large span in luminosity can be explained within the spin equilibrium scenario only if $B \propto L_{X}^{1 / 2}$ (under the reasonable assumption that $L_{X} \propto \dot{M}$ ). However, the sample used by WZ97 suffered two serious biases:

1. all bright systems have NS spin frequencies determined only from twin-kHz QPOs, which today is not considered a robust method;

2. in two cases the spin period was taken at twice the observed value to match the $\Delta v$ of twin-kHz QPOs.

If we remove the first bias by ignoring twin-kHz QPO sources, then the luminosities span less than one order of magnitude instead of two. By removing also the second bias by using the observed spin period (instead of twice its value), the spin periods span a range between 1.7 and $2.8 \mathrm{~ms}(362-589 \mathrm{~Hz})$. The difference between the minimum and maximum spin frequency therefore becomes more than twice the value used by WZ97. Therefore, there is no clustering of MXP spin frequencies over two orders of magnitude in luminosity as reported by WZ97.

We can go further and repeat the analysis of WZ97 on the enlarged sample of MXPs given in Table 1, with the spin periods spanning now an even broader range between 1.6 and $5.5 \mathrm{~ms}$ $(182-620 \mathrm{~Hz})$. Assuming each source is in equilibrium and the magnetic field is given by Equation (4), we see no convincing correlation $B \propto L_{X}^{1 / 2}$ (see Figure 1). The field at equilibrium has now a large spread which depends on the broad scatter in observed $v_{s}$. Figure 1 shows also that the field is not stronger for bright sources and weaker for faint sources as was inferred by WZ97 (compare with Figure 1 in WZ97). 

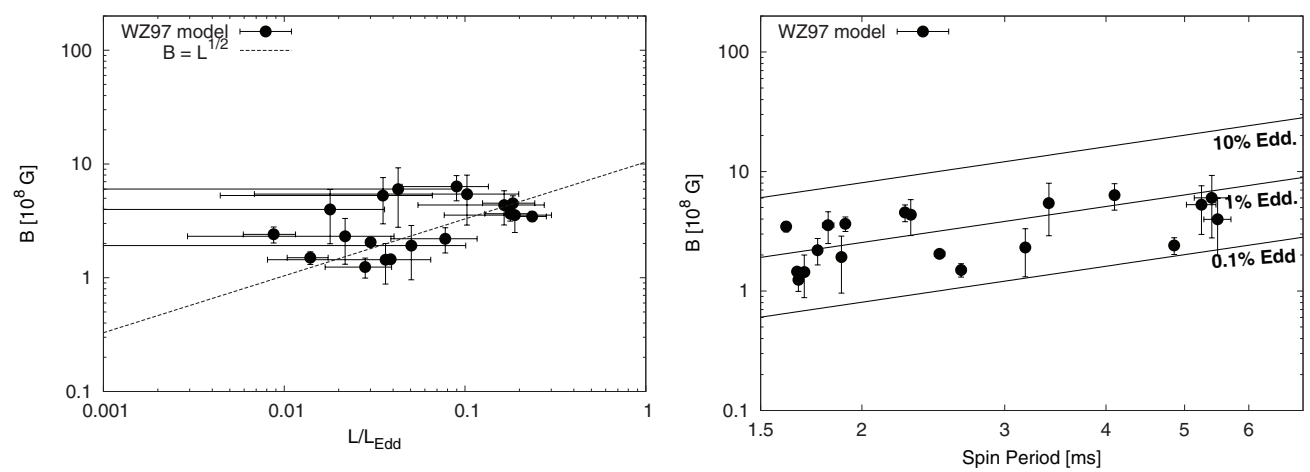

Figure 1. Left panel: inferred magnetic field at equilibrium vs. luminosity relation for MXPs as reported in Table 1. The black dots refer to magnetic fields inferred under the hypothesis of spin equilibrium $r_{m}=r_{\mathrm{co}}$. The Eddington luminosity for a $1.4 M_{\odot}$ is $L_{\mathrm{Edd}}=1.8 \times 10^{38} \mathrm{erg} \mathrm{s}^{-1}$. Right panel: inferred magnetic field at equilibrium vs. spin period. The plot uses the same sources and symbols as in the left panel. The three lines refer to luminosities of $0.1 \%, 1 \%$, and $10 \%$ Eddington.

\section{REALISTIC MODELS OF DISK/MAGNETOSPHERE INTERACTIONS}

The calculation in Section 3 does not incorporate the considerable uncertainties in calculating how angular momentum can be extracted from the star, which will strongly affect the spin equilibrium frequency. Here we revisit a more realistic model for the interaction between an accretion disk and the magnetic field, and demonstrate how these uncertainties can lead to a large variation in equilibrium spin periods for a given $B$ and $\dot{M}$.

The estimate for the magnetospheric radius given by Equation (1) does not consider the relative rotation between the star and the disk, and as such is only applicable when the disk is truncated well inside the corotation radius (that is, the magnetic field is rotating relatively slowly compared with the inner parts of the disk). As the disk moves outward, the relative rotation between the disk and star becomes critical in determining the location of $r_{m}$ and the disk structure. Once $r_{m}$ is very close to $r_{\mathrm{co}}$, Equation (1) fails completely, since now the magnetic field presents a centrifugal barrier to accretion. D'Angelo \& Spruit (2010) demonstrated that for $r_{m} \simeq r_{\mathrm{co}}$, the angular momentum added by the disk-field interaction can change the disk structure and lead to a disk truncated just outside $r_{\text {co }}$ without any accretion onto the star or outflow.

Calculating the spin rate in equilibrium for a given accretion rate is thus not as simple as equating Equation (1) with Equation (3), and requires a good understanding of the spindown torques, which are subject to large uncertainties. For the picture presented in D'Angelo \& Spruit (2010) the spindown torque comes from the interaction between the disk and field outside corotation. This leads to a spin-down torque of magnitude

$$
\dot{J}=\eta \frac{\Delta r}{r_{m}} \frac{\mu^{2}}{r_{m}^{3}},
$$

where $\mu=B^{2} R^{3}$ is the magnetic dipole moment, $\eta \sim 1$ is a dimensionless coefficient that quantifies the strength of the disk-field coupling, and $\Delta r / r_{m}<0.3$ indicates the radial extent of the coupling between the star and the disk. Both these parameters will be dependent on the detailed disk-field coupling and may vary between systems as a result of, e.g., the magnetic field inclination or the large-scale magnetic field configuration (D'Angelo \& Spruit 2011). An additional complication in predicting the amount of spin-down torque is the appearance of episodic cycles of accretion (Spruit \& Taam 1993; D'Angelo \& Spruit 2010, 2011), in which mass accumulates just outside $r_{c}$ and is periodically dumped onto the star. D'Angelo \& Spruit
(2011) have found that the net amount of spin-down in cyclic accretion can be up to $100 \%$ larger than steady accretion at the same accretion rate.

Other sources of angular momentum loss from the star can come from magneto-dipole radiation or stellar outflows. Magneto-dipole radiation may dominate the spin-down for sources in quiescence (Hartman et al. 2008, 2009), but is unlikely to dominate over the disk-field interaction while the star is in outburst. In contrast, a considerable amount of angular momentum could be expelled via a stellar wind, depending on how much mass is ejected in an outflow (Matt \& Pudritz 2005). Stellar winds have been observed in numerical simulations to carry away a large amount of angular momentum and could compete or even dominate over field-disk coupling as a source of angular momentum loss in the star (Romanova et al. 2009).

Finally, at high accretion rates, the coupling between the disk and field can also change the amount of angular momentum deposited onto the star from accretion (Andersson et al. 2005). In this case the inner parts of the disk become radiation-pressuredominated, and the spin equilibrium condition translates then into the relation

$$
B \propto\left(1-\frac{L_{X}}{L_{\mathrm{Edd}}}\right)^{-5 / 6} L_{X}^{1 / 2} v_{s}^{-7 / 6}
$$

(see Equations (38) and (45) in Andersson et al. 2005).

Summarizing the value of $\eta \Delta r / r$ can be different in different systems and this might introduce a spread of $B$ fields at equilibrium up to an order of magnitude. For bright sources this spread can increase up to three orders of magnitude because of the effect of radiation-pressure-dominated disks. Therefore, we would not expect to observe $B \propto L_{X}^{1 / 2}$ even for sources that were all spinning at the same frequency. We show this in Figure 2 where we have chosen a hypothetical accreting NS spinning at $730 \mathrm{~Hz}$ and a range for the parameter $\eta \Delta r / r$ in Equation (5) between 0.001 and 0.3 (D'Angelo \& Spruit 2011). We have also included the possibility that the disk becomes radiation-pressure-dominated as described in Andersson et al. (2005), with $\xi=0.3$ and 1 .

The allowed region in Figure 2 shows that a $B$ field of $\sim 10^{8} \mathrm{G}$ is already sufficient for an MXP spinning at $730 \mathrm{~Hz}$ at equilibrium regardless of the source luminosity. Furthermore, since $B \propto v_{s}^{-7 / 6}$, any accreting NS spinning above $730 \mathrm{~Hz}$ also requires fields of the order of $10^{8} \mathrm{G}$ or lower at equilibrium. Therefore, there is no need for bright systems like Sco X-1 to have larger $B$ fields at equilibrium than faint sources. 


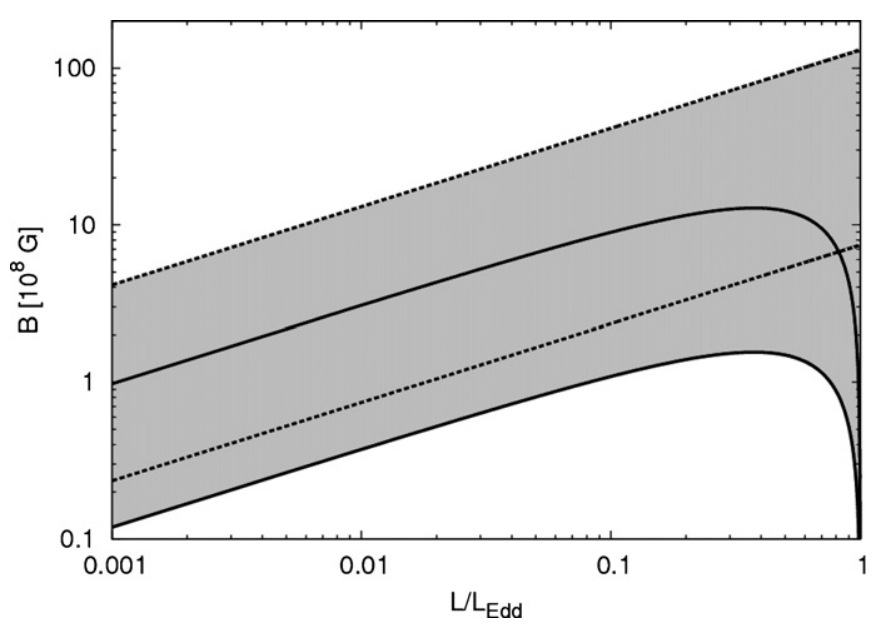

Figure 2. Allowed parameter space (shaded gray area) between the inferred magnetic field at equilibrium and the source luminosity for a hypothetical $730 \mathrm{~Hz}$ accreting NS. The top and bottom dashed lines are obtained by equating the spin-down in Equation (5) and the spin-up in Equation (2) and are calculated for $\eta \Delta r / r=10^{-3}$ and 0.3 , respectively. The solid lines refer to the radiationpressure-dominated disk solution of Andersson et al. (2005), with $\xi=0.3$ and 1 . For bright systems the inferred $B$ field at equilibrium has an uncertainty that spans almost three orders of magnitude.

\section{DISCUSSION}

In this paper, we have re-assessed the possibility that the apparent cutoff at $730 \mathrm{~Hz}$ in the spin distribution of accreting NSs may be due to these systems being at or close to spin equilibrium, as set by the disk/magnetosphere interaction. This possibility was examined in detail by WZ97, who concluded that such a scenario would require an unexpected correlation between the magnetic field of the star and the outburst luminosity. Their analysis was, however, biased by the relative scarcity of observations and by the inclusion in their sample of bright systems for which the spin frequency was inferred from the separation of the twin-kHz QPOs, a method which is now known to be unreliable (Méndez \& Belloni 2007). Nevertheless, the work of WZ97 did lead to the suggestion that the disk/magnetosphere interaction alone was insufficient to explain the spin-frequency distribution of accreting NSs, unless the $B$ field at equilibrium was correlated with the X-ray luminosity of the binaries. To avoid this correlation, Bildsten (1998) proposed that GWs may remove angular momentum at a rate sufficient to explain the observed spin distribution.

Repeating the analysis of WZ97 with the extended sample of systems available today and removing the twin- $\mathrm{kHz}$ QPO systems, we have shown that no strong correlation between the magnetic field strength and luminosity is required to explain the observed spin distribution. This is due to two main effects. First, the spread in observed spin frequencies has considerably increased since the work of WZ97, leading to a larger scatter in the values for the magnetic field strength required for equilibrium, even in the simplest disk/magnetosphere picture. On the other hand, the disk structure itself and the source of spin-down torque vary from system to system, leading to a considerable range of equilibrium $B$ for a given $v_{S}$ and $L_{x}$.

From an observational point of view, there is evidence that two of the MXPs may be close to spin equilibrium during the outburst (Haskell \& Patruno 2011), while the MXP IGR J00291+5934 (and to some extent XTE J1751-395; Riggio et al. 2011a) exhibits a saw-tooth-like behavior, with the spinup during the outburst nearly balanced by the spin-down during quiescence (Patruno 2010a; Papitto et al. 2011b; Hartman et al. 2011). This behavior suggests that these systems are in longterm spin equilibrium (Elsner et al. 1980; Lamb \& Yu 2005), a scenario which would be consistent with the inferred magnetic field of IGR J00291+5934 ( $\left.B \approx 2 \times 10^{8} \mathrm{G}\right)$ and XTE J1751-305 $\left(B \approx 4 \times 10^{8} \mathrm{G}\right)$.

Furthermore, Haskell \& Patruno (2011) have shown that GW emission is not consistent with the timing properties and quiescent luminosity of SAX J1808.4-3658 and XTE J1814338. Note that Ho et al. (2011) have suggested that GW emission may be required to explain the lack of observed systems with low spin rates and long orbital periods. This argument is, however, based on the assumption that the spin-up torque is of the simple form in Section 3 and it might be necessary to re-assess this with more realistic models.

The situation may be quite different for bright persistent sources like Sco X-1 and other persistent GX and Z sources (see van der Klis 2004 for a review on the source classification). For most of these sources the spin frequency of the NS is not known, and the high accretion rate would imply spin equilibrium, for the simple accretion torque of Section 3, well above the Keplerian break-up frequency for magnetic field strengths of the order of $B \approx 10^{8} \mathrm{G}$. Although it is possible that these systems do contain a sub-millisecond pulsar, this might be difficult to reconcile with the fact that the distribution of millisecond radio pulsars also appears to have a cutoff at around $700 \mathrm{~Hz}$ (Hessels et al. 2006). However, unlike X-ray pulsars, the sensitivity of radio surveys to sub-millisecond pulsars degrades with the unknown dispersion measure, which might partially explain why radio sub-millisecond pulsars have not been found so far (Hessels et al. 2007).

A second possibility is that these systems have reached spin equilibrium at $\sim 700 \mathrm{~Hz}$ with a stronger magnetic field in bright systems, as predicted by Konar \& Bhattacharya (1999). If the $B$ field is confined to the NS crust a positive correlation exists between the average mass accretion rate (which, unlike the outburst accretion rate, does not depend on the details of the disk instability model) and the final field strength. In this scenario, the mechanism responsible for the $B$ field decay is Ohmic dissipation in the NS crust, which is substantially accelerated with accretion. The accreted material also pushes the current producing the $B$ field deeper down to the crust-core interface, where resistivity is low and Ohmic dissipation becomes unimportant (see, e.g., Romani 1990).

An alternative scenario is that these bright systems may, in fact, be emitting GWs. The higher temperatures of these systems and the constant stream of accreted material make it likely that the star could sustain a mountain in the crust, due to compositional and heating asymmetries (Ushomirsky et al. 2000; Haskell et al. 2006) or that the magnetic field may be distorted by the accretion flow and support a substantial quadrupole (Melatos \& Payne 2005; Priymak et al. 2011).

However, a plausible minimal scenario is that, as discussed in Section 4, the spin-up torque is much weaker than the estimate in Section 3 at higher luminosities, while the magnetic field strength is still in the region of $B \approx 10^{8} \mathrm{G}$. This could either be due to the inner parts of the disk being radiation-pressure-dominated (Andersson et al. 2005) the disk/magnetosphere coupling being weaker (D'Angelo \& Spruit 2010), or to additional sources of spin-down, such as mass outflows (Romanova et al. 2009).

It would thus appear that the observed spin distribution is consistent with the notion that all systems are either at, or 
close to, spin equilibrium. This picture does not require a strong correlation between the magnetic field strength and the luminosity and is compatible with modest field strengths of the order $10^{8} \mathrm{G}$, with no need to assume much higher field strengths for any systems. This field strength is compatible with the $B$ field that is inferred from the quiescent spin-down of three MXPs.

A.P. acknowledges support from the Netherlands Organization for Scientific Research (NWO) Veni Fellowship, B.H. from the European Union via a Marie Curie IEF Fellowship. C.D. is supported by an NWO Vidi Fellowship.

\section{REFERENCES}

Andersson, N., Glampedakis, K., Haskell, B., \& Watts, A. L. 2005, MNRAS, 361,1153

Bildsten, L. 1998, ApJ, 501, L89

Chakrabarty, D. 2008, in AIP Conf. Proc. 1068, A Decade of Accreting Millisecond X-Ray Pulsars, ed. R. Wijnands, D. Altamirano, P. Soleri et al. (Melville, NY: AIP), 67

Chakrabarty, D., Morgan, E. H., Muno, M. P., et al. 2003, Nature, 424, 42

D'Angelo, C. R., \& Spruit, H. C. 2010, MNRAS, 406, 1208

D'Angelo, C. R., \& Spruit, H. C. 2011, MNRAS, 416, 893

Elsner, R. F., Ghosh, P., \& Lamb, F. K. 1980, ApJ, 241, L155

Hartman, J. M., Galloway, D. K., \& Chakrabarty, D. 2011, ApJ, 726, 26

Hartman, J. M., Patruno, A., Chakrabarty, D., et al. 2008, ApJ, 675, 1468

Hartman, J. M., Patruno, A., Chakrabarty, D., et al. 2009, ApJ, 702, 1673

Haskell, B., Jones, D. I., \& Andersson, N. 2006, MNRAS, 373, 1423

Haskell, B., \& Patruno, A. 2011, ApJ, 738, L14

Hessels, J. W. T., Ransom, S. M., Stairs, I. H., Kaspi, V. M., \& Freire, P. C. C. 2007, ApJ, 670, 363

Hessels, J. W. T., Ransom, S. M., Stairs, I. H., et al. 2006, Science, 311, 1901
Ho, W. C. G., Maccarone, T. J., \& Andersson, N. 2011, ApJ, 730, L36

Illarionov, A. F., \& Sunyaev, R. A. 1975, A\&A, 39, 185

Konar, S., \& Bhattacharya, D. 1999, MNRAS, 303, 588

Lamb, F., \& Yu, W. 2005, in ASP Conf. Ser. 328, Binary Radio Pulsars, ed.

F. A. Rasio \& I. H. Stairs (San Francisco, CA: ASP), 299

Lasota, J.-P. 2001, New Astron. Rev., 45, 449

Matt, S., \& Pudritz, R. E. 2005, ApJ, 632, L135

Melatos, A., \& Payne, D. J. B. 2005, ApJ, 623, 1044

Méndez, M., \& Belloni, T. 2007, MNRAS, 381, 790

Papaloizou, J., \& Pringle, J. E. 1978, MNRAS, 184, 501

Papitto, A., Ferrigno, C., Bozzo, E., et al. 2011a, ATel, 3556, 1

Papitto, A., Riggio, A., Burderi, L., et al. 2011b, A\&A, 528, A55

Patruno, A. 2010a, ApJ, 722, 909

Patruno, A. 2010b, arXiv:1007.1108

Pringle, J. E., \& Rees, M. J. 1972, A\&A, 21, 1

Priymak, M., Melatos, A., \& Payne, D. J. B. 2011, MNRAS, 417, 2696

Psaltis, D., \& Chakrabarty, D. 1999, ApJ, 521, 332

Riggio, A., Burderi, L., di Salvo, T., et al. 2011a, A\&A, 531, A140

Riggio, A., Papitto, A., Burderi, L., et al. 2011b, A\&A, 526, A95

Romani, R. W. 1990, Nature, 347, 741

Romanova, M. M., Ustyugova, G. V., Koldoba, A. V., \& Lovelace, R. V. E. 2009, MNRAS, 399, 1802

Spruit, H. C., \& Taam, R. E. 1993, ApJ, 402, 593

Ushomirsky, G., Cutler, C., \& Bildsten, L. 2000, MNRAS, 319, 902

van der Klis, M. 2004, arXiv:astro-ph/0410551

van der Klis, M., van Paradijs, J., Lewin, W. H. G., et al. 1996, IAU Circ., 6428,

van Paradijs, J., \& White, N. 1995, ApJ, 447, L33

Wagoner, R. V. 1984, ApJ, 278, 345

Watts, A. L., Krishnan, B., Bildsten, L., \& Schutz, B. F. 2008, MNRAS, 389, 839

White, N. E., Stella, L., \& Parmar, A. N. 1988, ApJ, 324, 363

White, N. E., \& Zhang, W. 1997, ApJ, 490, L87

Wijnands, R., van der Klis, M., Homan, J., et al. 2003, Nature, 424, 44

Yin, H. X., Zhang, C. M., Zhao, Y. H., et al. 2007, A\&A, 471, 381 